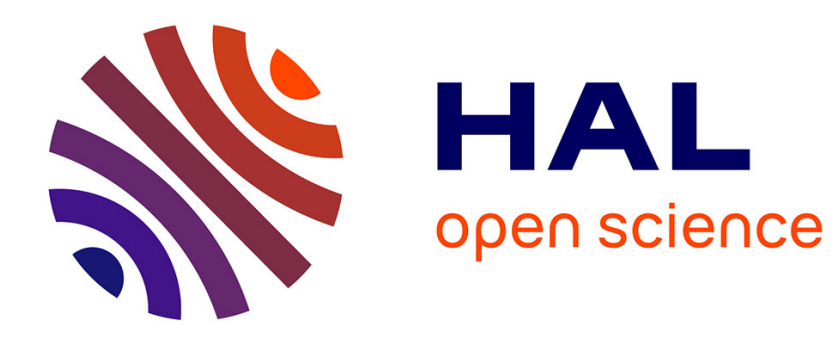

\title{
La préposition et la tradition grammaticale anglaise
}

Claude Guimier

\section{To cite this version:}

Claude Guimier. La préposition et la tradition grammaticale anglaise. Langages, 2007, 167 (3), pp.85-99. 10.3917/lang.167.0085 . hal-00012171

\section{HAL Id: hal-00012171 \\ https://hal.science/hal-00012171}

Submitted on 19 Oct 2005

HAL is a multi-disciplinary open access archive for the deposit and dissemination of scientific research documents, whether they are published or not. The documents may come from teaching and research institutions in France or abroad, or from public or private research centers.
L'archive ouverte pluridisciplinaire HAL, est destinée au dépôt et à la diffusion de documents scientifiques de niveau recherche, publiés ou non, émanant des établissements d'enseignement et de recherche français ou étrangers, des laboratoires publics ou privés. 


\section{La préposition et la tradition grammaticale anglaise}

Claude Guimier

Caen, CRISCO : FRE 2805

\section{Introduction}

L'existence même de la préposition, en tant que partie du discours, est bien établie en grammaire anglaise. Héritée de la próthesis grecque et de la prepositio latine, la préposition figure en bonne place dès les premiers ouvrages portant sur la langue vernaculaire. Cela ne saurait surprendre dans la mesure où ces ouvrages utilisent largement le système des parties du discours de la grammaire latine ${ }^{1}$. La préposition ne sera pas remise en cause par les générations suivantes de grammairiens, pas plus qu'elle ne l'est par les linguistes contemporains. Tout au plus peut-on rappeler la tentative de certains distributionnalistes américains pour libérer l'analyse grammaticale du carcan des parties du discours traditionnelles et catégoriser les unités de la langue en fonction de leur seule distribution syntagmatique. Chez Fries (1952), cette volonté de rupture se manifeste par le refus de dénommer chaque classe ; on reconnaît néanmoins dans son groupe $\mathrm{F}$ de mots fonctionnels la préposition traditionnelle. La préposition apparaît ainsi comme une catégorie syntaxique indispensable à la description de la langue anglaise. Toutefois, cette unanimité de façade ne doit pas masquer certaines difficultés. L'examen du traitement de la classe prépositionnelle fait apparaître des divergences et des problèmes récurrents, notamment au niveau de la définition de la classe et de sa délimitation. En d'autres termes, le concept que recouvre le métaterme de préposition ne semble pas stabilisé, ce qui fragilise le caractère opératoire de la catégorie.

Le paragraphe d'introduction au chapitre consacré aux prépositions dans Greenbaum \& Quirk 1990 (chap. 9) - manuel de grammaire universitaire contemporain - résume bien les propriétés traditionnellement attribuées à la catégorie :

Prepositions are a closed class of items [...] connecting two units in a sentence and specifying a relationship between them. For example :

(I don't like to) drink out of a cracked cup

(He was) very grateful for her help

The elderly man in the raincoat (looks ill)

In all three, the second unit is the prepositional complement, but in [1] the link is to a verb phrase, in [2] an adjective phrase, and in [3] a noun phrase. The sequence of preposition and its complement is known as a prepositional phrase [...]. [...] But [...] prepositional phrases can also function as adverbials, and the 'link' can then be between the prepositional complement and a whole clause. Compare, for example, the adverbial in [4]:

In a few minutes, we'll know the result of the blood test

Prepositions may be simple or complex, and in either case their complements are usually noun phrases; but they can also be nonfinite (-ing clauses) or nominal (wh-) clauses.

En résumé :

a) la préposition est un relateur qui établit un lien entre deux entités

b) le complément de la préposition est un groupe nominal (mais certaines propositions sont possibles)

c) le groupe prépositionnel dépend d'un autre constituant: syntagme verbal, adjectival ou nominal, ou proposition tout entière

d) la préposition appartient à une classe fermée

\footnotetext{
${ }^{1}$ Sur la place de la préposition dans les grammaires anglaises publiées jusqu'en 1800, voir Michaël 1970 (chap. 8 et Appendix I).
} 
e) la préposition peut être simple ou complexe

f) la préposition spécifie - il faut comprendre sémantiquement - le lien entre les deux entités mises en relation

La préposition se définit ainsi au travers d'un certain nombre de propriétés syntaxiques, morphologiques et sémantiques. Dans les lignes qui suivent, nous examinerons quelques-unes de ces propriétés et nous nous interrogerons sur leur pertinence en tant que critères permettant de distinguer la préposition d'autres catégories syntaxiques, notamment l'adverbe et la conjonction. Pour ce faire, nous nous appuierons essentiellement sur deux ouvrages de grammaire majeurs de l'anglais contemporain: d'un côté, Quirk et al., Contemporary Grammar of the English Language, Longman, 1985 (désormais CGEL 1985) qui présente un traitement de la préposition qui correspond largement aux traitements traditionnels ${ }^{2}$; de l'autre, Huddleston \& Pullum et al., The Cambridge Grammar of the English Language, CUP, 2002 (désormais CGEL 2002) qui, tout particulièrement dans le domaine des prépositions, revendique une rupture explicite avec la tradition ${ }^{3}$. Nous ne retiendrons que quelques traits saillants traditionnellement attachés aux prépositions - la fonction de relation, la nature et la nécessité d'un complément, leur valeur sémantique - et nous nous interrogerons sur le bien-fondé des deux types d'approche.

\section{La préposition est-elle un relateur ?}

Dire que la préposition est un mot de relation implique que partout et toujours elle intervient entre un avant et un après, selon le schéma $X$ prép. $Y$. Il ne peut toutefois s'agir d'une propriété distinctive puisque d'autres classes syntaxiques regroupent des éléments qui ont un rôle de connecteur : pronoms relatifs, conjonctions, adverbes conjonctifs. Par ailleurs, une connexion entre deux unités peut être établie par d'autres moyens, par exemple le recours aux $\operatorname{cas}^{4}$. Ceci reste vrai de l'anglais moderne, qui a conservé un cas génitif, soumis à un certain nombre de contraintes, et qui alterne souvent avec des constructions prépositionnelles : the fisherman's cottage / the cottage of the fisherman; an old people's home / a home for old people. Une relation de connexion peut également être établie sans marqueur particulier et l'anglais fait notamment un recours abondant aux agrégats nominaux qui permettent d'établir des relations sémantiques variées entre des unités nominales : the corner table vs. the table in the corner; a Dickens novel vs. a novel by Dickens. On pense également au "complément d'attribution », introduit, ou non, au moyen d'une préposition, en fonction de sa position : I gave him the book / I gave the book to him; ou encore à certaines constructions binominales dénotant une identification entre les deux noms, avec préposition (the county of Kent), ou sans marque relationnelle (The River Thames). Enfin, le recours à la préposition semble parfois facultatif: We stayed here for three months / We stayed here three months; I'm not certain of what I'm supposed to do / I'm not certain what I'm supposed to do. Si la préposition doit se définir par son caractère relationnel, cela ne peut constituer qu'une propriété nécessaire, mais non suffisante, de cette classe de mots.

\footnotetext{
${ }^{2}$ Ce qui ne signifie pas que CGEL 1985 soit globalement une grammaire traditionnelle. Elle se démarque de celles-ci par son recours à un large corpus (Survey of English Usage) et elle intègre certains acquis de la linguistique moderne. Son traitement des prépositions néanmoins n'est pas fondamentalement différent de celui des grammaires traditionnelles.

3 "This book employs a definition of the category of prepositions that is considerably broader than those used in traditional grammars of English. [...] In this book, in keeping with much work in modern linguistics, we adopt a significantly different conception of prepositions." (CGEL 2002, 598)

${ }^{4}$ Les prépositions ont longtemps été considérées comme des formes supplétives des cas.
} 
Ce caractère relationnel, mis en avant par CGEL 1985 (p. 657), fait rarement l'objet d'une réflexion théorique : la préposition est-elle à l'origine de la relation entre $\mathrm{X}$ et $\mathrm{Y}$ ou comble-telle une relation pré-existante, par exemple en lui donnant un contenu notionnel ? S'agit-il d'une relation syntaxique ou d'une relation sémantique ? Les deux termes de la relation sontils nécessairement instanciés ? La relation est-elle orientée ?

Le rôle de relateur de la préposition est manifeste dans a knock on the door, banned from the club, talk to the teacher, et il n'est pas surprenant que ce soient des exemples de ce type qui sont cités en premier par Greenbaum \& Quirk. Toutefois, ce rôle est déjà moins apparent en $[4]^{5}$ : pour ces auteurs, la préposition opère ici un lien entre le complément prépositionnel et la proposition entière, impliquant ainsi que la relation avant / après n'est pas toujours reflétée par le linéaire. Se trouve ainsi posé le problème du détachement des syntagmes prépositionnels : dans We'll know the result of the blood test in a few minutes, la préposition introduit un complément intégré à la proposition et son avant se trouve bien à sa gauche dans le linéaire. Admettre qu'on a le même type de liaison en [4], c'est reconnaître que cette liaison n'est pas d'ordre syntagmatique, mais tout au plus sémantique.

La question se pose en outre de savoir si le complément constitue toujours l'après de la relation. Dans un exemple tel que To my regret, he did not accept our offer, sémantiquement on peut admettre que c'est le complément de la préposition qui constitue le terme $\mathrm{X}$, en tant que prédicat nominalisé ayant pour second argument la proposition (cf. I regret that he did not accept our offer). Il en va de même dans my scamp of a husband (= my husband is a scamp) ou dans the whiteness of her skin (= her skin is white). Dans In other words, she was sentenced to solitary confinement, il est admis que le groupe prépositionnel fonctionne comme un adverbe d'énonciation et si la préposition établit une relation, l'élément $\mathrm{X}$ ne trouve pas d'expression linguistique mais correspond à l'acte d'énonciation lui-même, comme le suggère une paraphrase maladroite telle que: I now say in other words that she was sentenced to solitary confinement. Définir la préposition comme un mot de liaison apparait ainsi comme peu opératoire syntaxiquement et sémantiquement car, outre son caractère non discriminant, ce critère perd de sa pertinence tout particulièrement lorsque le groupe prépositionnel est en position détachée. Plus important dans cette configuration semble être le rôle translatif de la préposition, mis en avant par Tesnière $(1966,82)$ : la préposition permet de construire un groupe prépositionnel qui pourra assumer une fonction que le complément de la préposition ne peut assurer par lui-même ; soit une fonction adverbiale, avec tous les degrés possibles d'intégration à l'énoncé, depuis l'intégration maximale en fonctionnement intraprédicatif (She did it with great care) jusqu'à l'intégration minimale en fonctionnement exophrastique (to my knowledge, in other words dans les ex. cités) ${ }^{6}$; soit une fonction adjectivale lorsque le groupe prépositionnel est incident à un support nominal (a man without scruples; a woman with child; adviser to the president). Toutefois, dans les constructions telles que his anger over the matter, keen on football, their survival depends on him, la préposition introduit un argument du nom, de l'adjectif ou du verbe, et, à moins de considérer a priori que tout groupe prépositionnel est un adverbial - ce que suggère CGEL 1985 au chapitre 2 avant de revenir à une analyse en termes de complémentation au chapitre 16 - force est d'admettre que le rôle essentiel de la préposition est ici d'établir le lien entre un élément prédicatif et l'un de ses arguments. Apparaît ainsi la difficulté à laquelle se heurte toute tentative de caractérisation unitaire de la préposition.

Le rôle de relateur n'est pas mis en avant par CGEL 2002 qui retient par contre celui de la préposition comme tête du groupe prépositionnel. Deux types d'arguments sont avancés : (a)

\footnotetext{
${ }^{5}$ Est-ce la raison pour laquelle le mot link est utilisé avec des guillemets ?

${ }^{6}$ Sur les différents types de fonctionnements adverbiaux, v. Guimier 1996. Certains groupes prépositionnels exophrastiques sont lexicalisés et considérés comme des adverbes authentiques : in fact, of course.
} 
à l'instar des autres groupes syntaxiques (groupes nominaux, adjectivaux, verbaux), certains groupes prépositionnels peuvent recevoir des pré-modifieurs, notamment de degré : very much in control of things, shortly after midnight, just inside the penalty area ${ }^{7}$; (b) à l'instar des autres têtes de syntagmes, les prépositions peuvent recevoir des compléments de divers types. Cette thèse se heurte à l'opinion établie selon laquelle le complément de la préposition est normalement de nature nominale.

\section{Le complément de la préposition est-il nécessairement nominal ?}

On a vu que cette opinion couramment admise était modulée par Greenbaum et Quirk (1990) qui admettent qu'une préposition puisse avoir pour compléments certains types de propositions. CGEL 1985 (p. 658) précise que seules les propositions en that et les infinitives sont exclues comme compléments prépositionnels. Ces traits négatifs constituent une propriété définitoire de la catégorie. Cette propriété ne vaudrait toutefois que pour les prépositions «centrales », certaines prépositions «marginales» contrevenant à la règle (instead of to strip things off). Or il est difficile d'admettre que in constitue une préposition marginale ; elle accepte pourtant comme complément une complétive en that: I'm lucky in that I have four sisters ${ }^{8}$. Faire de la séquence in that une conjonction complexe (loc. cit.) n'a aucune justification linguistique (in introduit un argument de l'adjectif lucky, tout comme dans I'm lucky in love), si ce n'est celle de sauver le principe édicté au départ.

Par ailleurs, la plupart des auteurs sont obligés d'admettre qu'une préposition a la faculté d'introduire des éléments non nominaux: adjectifs (in earnest, in short, is that anywhere near correct?), adverbes (till now, by then, until recently), groupes prépositionnels (from behind the counter, in between the trees, till after the holidays). Le dogme du complément nominal conduit certains grammairiens à considérer que l'adjectif ou l'adverbe sont alors logiquement équivalents à des noms. Pour Sweet $(1891,136)$ : in short $=$ in few words ; till now $=$ till the present time. Faut-il alors considérer que ever correspond au nom eternity dans for ever et admettre une forme d'ellipse dans since before the war (= since the days before the war) ? Et en quoi le complément de la préposition est-il nominal dans for good ou dans next to impossible?

De surcroît, la préposition peut accepter un groupe prédicatif averbal comme complément. Jespersen (MEG V, chap. 5) cite de nombreux exemples de "nexus » fonctionnant comme régimes de préposition, notamment, mais pas exclusivement, avec la préposition with ${ }^{9}$ :

Why should I wash my hands if I am quite comfortable with them dirty?

I can't write with you standing there.

Her heart was always pricked with remorse for hurt inflicted.

With a sensation of every limb gone weak.

Dans ces exemples, le complément de la préposition est une relation prédicative comportant un support nominal ou pronominal et un prédicat adjectival ou participial.

L'analyse traditionnelle admet qu'une préposition puisse avoir pour complément une proposition gérondive, ou une proposition en wh-, mais elle ne reconnaît pas la possibilité pour une préposition d'admettre comme complément d'autres types de proposition. Une telle

\footnotetext{
${ }^{7}$ Exemples cités par CGEL 2002, 599. Cet argument est également avancé par Melis 2003, 26.

${ }^{8}$ D'autres prépositions gouvernent une complétive : except (that $P$ ); save (that $P$ ).

${ }^{9}$ Les exemples suivants sont empruntés à Jespersen. Melis $(2003,17)$ montre également que la préposition avec accepte comme complément « une structure de type phrastique. »
} 
attitude se traduit par l'éclatement catégoriel de certaines formes. On va opposer par exemple un for prépositionnel (I'm waiting for her; Thank you very much for having us to tea) et un for analysé comme subordonnant lorsqu'il introduit une infinitive (It would be better for them to leave at once.

La solution de CGEL 2002 - les prépositions sont des têtes de syntagme qui sélectionnent différents types de complément - a l'avantage de présenter un traitement unitaire des catégories syntaxiques majeures : verbe, nom, adjectif et préposition. Elle a pour conséquence une rupture revendiquée par rapport à la tradition et se traduit par un élargissement considérable de la classe. La grammaire traditionnelle se trouve confrontée au problème de l'homonymie de certaines formes, qui se trouvent éclatées entre diverses classes, éclatement que le sens des unités concernées ne justifie pas. Sur ce plan, CGEL 1985 s'en tient à la tradition stricte et, par exemple, analyse before comme une conjonction dans before she arrived et comme une préposition dans before her arrival. L'analyse grammaticale a tout à gagner en considérant qu'on a affaire à la même unité dans les deux cas : l'élément stable est la préposition, c'est la nature du complément qui varie. Nul ne peut contester l'analogie de fonctionnement (rappelée par CGEL 2002, 600) entre verbe et préposition :

\section{I remember the accident \\ He left after the accident}

\section{I remember you promised to help He left after you promised to help}

S'il est admis que remember ne change pas de nature selon la nature du complément régi, il n'y a aucune raison de penser qu'il puisse en être autrement dans le cas de after ${ }^{10}$. Poussant la logique jusqu'à son terme, les auteurs de CGEL 2002 ne conservent dans la classe des conjonctions (plus précisément des subordonnants) que les cinq unités suivantes : that (introducteur de complétive), whether et if lorsqu'il est substituable à whether (Ask him if / whether he minds), for et to introducteurs d'infinitives. Cette solution radicale ${ }^{11}$ présente de multiples avantages, notamment au niveau de la simplification et de la cohérence de l'analyse. Elle règle définitivement la question du statut d'un certain nombre d'unités telles que than qui donnent lieu à d'interminables discussions sur leur nature (conjonction dans He is bigger than I am, préposition dans He is bigger than me, mais également dans He is bigger than I, à moins qu'il faille voir ici une construction elliptique, auquel cas than y conserverait son statut de conjonction). Mais elle ne permet pas d'éviter toute forme d'éclatement. Dans CGEL 2002, for et to sont prépositions dans la majorité de leurs emplois mais, nous l'avons vu, subordonnants lorsqu'ils introduisent une proposition infinitive ${ }^{12}$. When est analysé comme mot interrogatif dans When is she leaving? (op. cit., 905), mot relatif dans at a time when I was living alone (op. cit., 1051), mais comme préposition dans His heart sank when he heard the news (op. cit., 698). Or dans tous ces emplois, on peut argumenter que when est un adverbe temporel et donc un adjoint pourvu, comme les autres mots en wh-, d'une fonction au sein de sa proposition. Et ce, contrairement à after, qui lui a bien un fonctionnement de type prépositionnel quels que soient ses emplois. Le regroupement proposé par CGEL 2002 n'est donc pas pleinement opératoire et les auteurs reconnaissent (p. 612) que les prépositions

\footnotetext{
${ }^{10}$ Pour un raisonnement analogue, v. Jespersen 1924, 89.

${ }^{11}$ Des solutions proches ont été proposées pour le français : pour Le Goffic (1993, 548-550), les conjonctions et locutions conjonctives sont des adverbes (quand, comme, si), des groupes adverbiaux (ainsi que, alors que, encore que), des groupes prépositionnels (après que, excepté que, en sorte que) ou des groupes nominaux (chaque fois que, le temps que).

${ }^{12}$ Sur les raisons de cette analyse, v. CGEL 2002, 1182-1184. Le to infinitival a toujours fait problème : il est souvent considéré de façon ad hoc comme constituant une particule inclassable (CGEL 1985, 67-68). Certains en ont fait un modal (cf. CGEL 2002, 1186 pour une réfutation de cette analyse) mais rares sont ceux qui en font une préposition (pour une exception, v. Duffley 1992, 144).
} 
prototypiques admettent pour complément un groupe nominal. Ailleurs (p. 603), il est question de prépositions centrales sans que soit précisé si ce sont les mêmes que les prépositions prototypiques. Dans cette grammaire, comme dans beaucoup d'autres, les notions de centralité et de prototypie sont utilisées sans faire l'objet d'un questionnement théorique. Par ailleurs, le problème de l'enchâssement d'une sous-phrase à une phrase matrice n'étant pas explicitement posé, la distinction entre préposition et subordonnant ne trouve finalement pas de solution satisfaisante.

\section{Le complément de la préposition est-il nécessaire ?}

Admettre que la préposition est un relateur implique qu'elle est nécessairement suivie d'un complément. Le problème typiquement anglais de la "préposition différée » (traduction littérale de " deferred preposition »), illustré par des exemples tels que The car she arrived in, He was made fun of, Who were you talking to?, ne constitue pas un contre-argument. Toutes les analyses s'appuient sur le fait que ces configurations apparaissent dans des schémas syntaxiques non canoniques; par exemple dans des relatives, des passives, des interrogatives. Cette opposition entre schémas canoniques et schémas non canoniques est inhérente à l'analyse grammaticale et prend diverses formes selon le cadre théorique retenu, mais aucune grammaire ne semble pouvoir s'en passer. L'opposition est longuement justifiée dans CGEL 2002 (p. 46-47), manuel qui, malgré le souci qu'il a de se démarquer de l'analyse traditionnelle sur de nombreux points, analyse les relatives (et les autres subordonnées) à partir de schémas de phrases simples, les phrases passives à partir des phrases actives, les interrogatives à partir des affirmatives. De fait, c'est l'unique moyen permettant de rendre compte de l'absence effective d'un complément à droite de la préposition dans ces phrases : ce complément est présent dans la construction canonique de base, il est déplacé dans la construction non canonique. Au passage, on relèvera le caractère inadéquat de l'étiquette « deferred preposition » (CGEL 1985, 663), qui laisse entendre que l'arrivée de la préposition a été retardée. L'expression «stranded preposition" (CGEL 2002, 58) - littéralement «préposition laissée en rade » - rend mieux compte du fait que la préposition conserve sa place de base mais qu'elle est « dégagée » de son complément ${ }^{13}$, lequel, extrait de sa position canonique pour occuper une autre position, est récupérable dans le contexte avant.

Plus problématique est le cas des " phrasal verbs » ou « verbes à particules adverbiales ». On sait que la description traditionnelle considère ces séquences comme des verbes composés, transitifs ou intransitifs. Ces particules sont, pour la plupart d'entre elles, homonymes de prépositions. Une telle analyse conduit là aussi à un éclatement au niveau de la catégorisation, une même forme ayant tantôt le statut d'adverbe, tantôt celui de préposition :

\section{Let me take that piece of thread off your skirt (prép.)}

The gentleman took his hat off / took off his hat (adv.; verbe composé adverbial transitif)

The plane took off(adv.; verbe composé adverbial intransitif).

Les grammairiens ont mis en évidence un certain nombre de tests qui justifient cette dichotomie, la même unité ayant un comportement différent dans l'un et l'autre cas, notamment au niveau de la position du complément nominal : celui-ci peut précéder ou suivre

\footnotetext{
13 La terminologie française a longtemps utilisé l'expression « rejet de la préposition» pour décrire ce phénomène. Or, la préposition conserve sa place de base dans le schéma canonique n'est donc pas " rejetée ». L'expression «dégagement de la préposition» proposée par Cherchi $(1986,61)$ a le mérite de décrire précisément le phénomène.
} 
la particule adverbiale ${ }^{14}$ mais il est obligatoirement à droite de la préposition. Jespersen (1924, 88) faisait déjà remarquer qu'une telle distinction était non pertinente et qu'il serait beaucoup plus « naturel » d'inclure dans une seule et même classe, celle des «particules », les unités telles que on dans put your cap on et put your cap on your head, ou down dans he falls down et he falls down the step. Toutefois, il inclut également dans cette classe l'ensemble des conjonctions, adverbes et interjections de la grammaire traditionnelle. Cette volonté d'unification enlève toute pertinence à la catégorie car celle-ci se trouve définie négativement comme constituée de toutes les unités qui ne sont ni substantifs, ni adjectifs, ni pronoms, ni verbes, leur seul point commun étant est leur indéclinabilité. Par ailleurs Jespersen ne prend pas en compte les « verbes composés » à sens non compositionnel (give in, drink up, come by, etc.).

Comme on pouvait s'y attendre, l'expression «phrasal verb» est rejetée par les auteurs de CGEL 2002 (p. 274). Dans cet ouvrage, les «particules adverbiales » sont des « prépositions intransitives », c'est-à-dire sans complément. Une préposition telle que off a un fonctionnement transitif dans She jumped off the wall (et dans ce cas le complément pronominal suit obligatoirement la préposition : she jumped off it / *she jumped it off) et un fonctionnement intransitif dans she took the label off / she took off the label (et dans ce cas, le complément pronominal précède obligatoirement la préposition : she took it off / *she took off it).

Admettre l'existence de prépositions intransitives va à l'encontre de la conception de la préposition comme relateur, mais nous avons vu que ce trait n'est pas proprement définitoire de la catégorie. Cela est par contre congruent avec une définition de la préposition comme tête de syntagme. Comme les autres types de syntagmes, le syntagme prépositionnel peut être réduit à son élément tête. On remarquera néanmoins que cette réduction ne correspond pas à un mode de fonctionnement unique. En particulier, l'intransitivité de la préposition est relative lorsque son complément est directement récupérable dans le contexte avant (on peut alors parler de préposition « orpheline ») :

\section{He cleared the table and pulled the cloth off (= pulled the cloth off the table)}

ou récupérable situationnellement :

\section{I didn't put sugar in (= in my coffee)}

Elle est par contre absolue lorsque nul complément ne peut être récupéré :

She gave in / He went on / We're saving up / I'll be glad to see this year out / The director told Peter off for being late.

Il est possible de mettre en évidence des cas intermédiaires : si, pour reprendre l'exemple de Jespersen, put a cap on peut être mis directement en relation avec put a cap on one's head, quel complément restituer dans put a coat on (= on oneself? ; on one's body? ; on one's back?). On a le sentiment que on gouverne bien un terme repère, mais que celui-ci n'est pas restituable. La plupart des prépositions intransitives voient ainsi leurs emplois osciller entre ces deux pôles : intransitivité absolue ou relative. L'existence d'un tel continuum (non mis en évidence par CGEL 2002) justifie largement le regroupement des « particules adverbiales »

\footnotetext{
${ }^{14}$ Cette propriété caractérise la classe ; elle ne vaut pas nécessairement pour chaque construction particulière : I must brush up my English before I go to London (?*brush my English up); The committee let the report through (? *let through the report).
} 
dans la classe des prépositions, les cas d'intransitivité absolue pouvant probablement s'expliquer par des phénomènes de métaphorisation et de figement à partir de configurations dans lesquelles la «particule» régit au départ un complément, fût-il implicite. Syntaxiquement, ces prépositions intransitives n'occupent d'ailleurs pas la position des adverbes ${ }^{15}$ :

\section{Bring in the stool from the bathroom / *Bring here the stool from the bathroom It swallowed up the two men / *It swallowed completely the two men}

CGEL 2002 (p. 613-616) rompt avec la tradition sur un autre point en incluant dans la classe des prépositions un ensemble relativement large de formes à valeur spatio-temporelle et habituellement reconnues comme des adverbes. Quelques exemples : abroad, ashore, aside (et autres formes en $a$-), here, there, where, east (et les autres points cardinaux), home, downhill, downstairs, indoors, underground, backward(s), inward(s) (et les autres formes en - ward(s)), now, then, beforehand, etc. Il s'agirait de prépositions qui ne régissent pas de complément. Les arguments retenus sont essentiellement de deux ordres : (a) la plupart de ces formes, comme les autres prépositions qui acceptent des emplois intransitifs, peuvent servir de complément de localisation pour be : he is in, behind / he is downstairs, there, home, etc. ; ou de complément de destination pour les verbes de mouvement : they went in, behind / they went downstairs, there, home, etc. ${ }^{16}$; (b) certaines peuvent être prémodifiées par right ou straight, à l'instar de quelques prépositions centrales : they are right downstairs; we went straight indoors.

Une telle recatégorisation a pour effet de réduire l'hétérogénéité bien connue et souvent dénoncée de la catégorie adverbe. Mais, inversement, ne génère-t-elle pas une forme d'hétérogénéité au niveau de la catégorie préposition? Si catégoriser, c'est regrouper dans une même classe des éléments qui se caractérisent par un ensemble de propriétés communes, il semble a priori difficile de regrouper des unités telles que now et over, home et in, downstairs et at. En fait, la plupart des unités recatégorisées se comportent moins comme des prépositions que comme des syntagmes prépositionnels (He stayed in his room / He stayed indoors) et si certaines prépositions en emploi intransitif peuvent apparaître dans la même position, c'est qu'elles sont alors véritablement « orphelines » et qu'un complément implicite leur est associé (He stayed in (his room)). La morphologie de certaines de ces formations montre qu'il s'agit de groupes prépositionnels condensés : une préposition est encore visible à l'initiale de formations telles que indoors, upstream ou overhead, mais aussi de aboard ou ahead où le «préfixe » $a$ - est historiquement une forme affaiblie de la préposition on. Autre point commun avec les groupes prépositionnels, ces formations peuvent apparaître comme sujets d'un verbe: In March suits me fine; During the vacation is what we decided ${ }^{17}$ vs. Indoors is dryer and less draughty; Downstairs is where the game room is located, Now would be a good time to do it. Une préposition authentique n'a pas cette capacité : *In is dryer and less draughty. Pour ces raisons, la description traditionnelle, qui considère que la plupart de ces formations sont des adverbes susceptibles, à l'instar des groupes prépositionnels,

\footnotetext{
${ }^{15}$ Exemples empruntés à Biber et al., 1999, 78 ; ce qui n'empêche pas cette grammaire de continuer à utiliser l'expression «particule adverbiale ». La possibilité de fonctionnement prépositionnel intransitif dépasse le cadre des «phrasal verbs ». Dans Ten days on the scale of disaster still grows, la préposition on, à laquelle on ne peut adjoindre de complément, implique néanmoins un repère temporel, en l'occurrence dans cet exemple le 26 décembre 2004, date du tsunami dans l'Océan Indien.

${ }^{16}$ A ce titre, ces formes se comportent différemment des adverbes en -ly: he is there / *he is locally; he went there / *he went locally.

${ }^{17}$ Exemples cités par CGEL 1985, 658, qui ajoute : « such nominal uses can be viewed as related to sentences that have been restructured so as to leave only the adverbial prepositional phrase : (To meet) in March ... ; (Meeting) during the vacation ...".
} 
d'assumer une fonction circonstancielle, apparaît préférable car dotée d'un pouvoir de généralisation plus grand. On pourrait montrer que d'autres unités considérées comme prépositions par CGEL 2002 sont simplement des substantifs en fonction circonstancielle (go home, east, etc.). L'élargissement de la classe trouve ici ses limites car les arguments avancés sont contrebalancés par autant de contre-arguments. Il semble préférable dans ces conditions de suivre la tradition et de reconnaître la transitivité - au moins potentielle - comme un trait définitoire de la préposition ${ }^{18}$, ce qui permet d'inclure dans la classe les «particules adverbiales $\gg$ mais non les adverbes spatio-temporels ${ }^{19}$.

\section{Les prépositions sont-elles fondamentalement spatiales?}

L'idée que les prépositions dénotent fondamentalement des relations spatiales n'est pas nouvelle. Elle est avancée dans les grammaires classiques ${ }^{20}$, mais ce trait est rarement mis en avant par les grammairiens du $19^{\text {ème }}$ et même du $20^{\text {ème }}$ siècle, si bien qu'on ne peut pas dire qu'il appartient à la tradition grammaticale anglaise. Nesfield $(1898,177-181)$ par exemple se borne à énumérer un certain nombre de valeurs propres à chaque préposition. Sweet (1891, 139) ne s'étend guère sur le sujet, se contentant d'affirmer que les différents sens des prépositions peuvent se ranger en trois rubriques : espace, temps et relations abstraites, une même préposition pouvant relever des trois domaines. L'approche de CGEL 1985 n'est pas fondamentalement différente. Après avoir souligné la multiplicité des relations prépositionnelles sur le plan sémantique, l'ouvrage met en avant les emplois spatiaux et temporels au motif que ce sont les plus faciles à décrire systématiquement. Le problème de la polysémie des prépositions est à peine posé et seulement pour les prépositions spatiales : «Many place prepositions have abstract meanings which are clearly related, through metaphorical connection, to their locative uses » (op. cit., 685). Le processus métaphorique lui-même n'est pas analysé. Pour le reste, CGEL 1985 opère dans la plus pure tradition des grammaires anglaises, et présente un catalogue des emplois des principales prépositions. Il s'agit du recensement le plus complet que l'on puisse trouver dans un manuel et à ce titre son utilité est incontestable, même s'il ne permet pas d'avoir une vue unitaire de chaque préposition, à supposer qu'une telle vue soit possible, et même légitime sur le plan linguistique.

CGEL 2002 se démarque également de l'approche traditionnelle dans son traitement sémantique des prépositions. Fortement influencé par la sémantique cognitive ${ }^{21}$, ce manuel défend la thèse de la primarité du spatial : «Most of the central prepositions in English (or any language) have meanings that are clearly locational in origin. More specifically, they concern either spatial location or change of location, or extensions of those notions into the dimension of time, or notions derived more broadly from them through other metaphors" (op. cit., 647-648). Il s'agit d'une position communément défendue à l'heure actuelle, non seulement par les cognitivistes mais également par des linguistes se situant dans des perspectives plus fonctionnalistes ou énonciativistes. Groussier (1997) défend ardemment cette position, mais non sans précautions : «il est évidemment tout à fait exclu de prétendre démontrer que, dans les langues dotées de prépositions, toute expression de relations au

\footnotetext{
${ }^{18}$ Sur la non existence de prépositions intransitives, v. Tremblay 1999, 178.

${ }^{19}$ Certes la catégorie verbale regroupe des unités totalement intransitives à côté d'autres qui sont transitives, mais les critères morphologiques sont déterminants pour le verbe alors qu'ils sont non pertinents pour la préposition.

20. Harris 1751 (book II, chap. 3) considère que les prépositions expriment primitivement des rapports de situation mais que, par métaphore, elles finissent par exprimer des rapports intellectuels. Voir aussi Murray $1795,77-78$.

${ }^{21}$ Pour une analyse des prépositions de l'anglais dans cette perspective, v. par exemple Tyler \& Evans 2003.
} 
moyen de prépositions soit l'expression de relations spatiales, tout autant que d'attribuer, en synchronie, aux sens spatiaux des prépositions, un quelconque rôle dominant : primarité n'est pas primauté $»^{22}$ (op. cit., 221). Elle montre que la plupart des prépositions des langues indoeuropéennes dérivent de formes anciennes qui ont d'abord eu des emplois spatiaux. On touche ici au fond du problème. La plupart des grammaires - et CGEL 2002 n'y échappe pas entretiennent une confusion terminologique, lourde de conséquences, entre sens primaire d'un côté et sens fondamental ou de base de l'autre. Parler de sens primaire, c'est se situer dans une perspective diachronique et prendre en compte l'étymologie du marqueur prépositionnel. Parler de sens de base, c'est (implicitement, car cela n'est pas clairement dit), se situer dans une perspective synchronique et, face à la polysémie de la préposition, prendre un sens intuitivement considéré comme plus fondamental ou prototypique (souvent parce qu'il semble le plus fréquemment réalisé) et lui accorder un rôle prééminent. CGEL 2002 utilise diverses expressions qui entretiennent un certain flou relatif à la perspective adoptée, diachronique ou synchronique. Ainsi si la préposition at se voit attribuer « a core lexical meaning » (p. 654), la préposition from est, elle, dotée de " a basic meaning " (p. 656) et of d'un " original basic locative meaning » (p. 658). Le point de vue semble clairement diachronique pour of, mais pas pour at et from. Et quel est le point de vue adopté pour with, considérée comme non fondamentalement spatiale puisque son «most basic meaning» (p. 661) est la notion d'accompagnement, alors qu'il est bien connu que, en vieil anglais, with a toutes sortes d'emplois clairement spatiaux (glosés par by, near, against, beside, through, etc.) ?

On voit ainsi les limites d'une approche de type localiste. Outre qu'une telle approche ne peut concerner que certaines prépositions considérées comme " prototypiques » (CGEL 2002, 647), et laisse de côté quantité d'unités pourtant étiquetées prépositions, elle ne se justifie pas nécessairement si l'on adopte une perspective purement synchronique. La valeur spatiale en effet, tout autant que les autres valeurs, est une valeur construite en discours et qui dépend largement de l'environnement co-textuel et situationnel. En d'autres termes, la valeur spatiale de to dans He went to Paris, sa valeur temporelle dans He worked from morning to evening et sa valeur résultative dans They beat him to death sont toutes trois dépendantes de l'environnement co-textuel. Faire appel à la notion de "context-induced reinterpretation », comme on le fait parfois lorsque la préposition n'a pas son sens spatial ${ }^{23}$, revient, en synchronie, à privilégier une valeur particulière pour en dériver les autres. On peut argumenter que le seul processus à l'œuvre est un processus de «context-induced interpretation », le co-texte étant déterminant dans tous les cas, quelle que soit la valeur en emploi de la préposition. Il ne s'agit pas de nier l'existence d'une valeur intrinsèque, attachée, en langue, à la préposition. Mais cette signification, qui sera nécessairement abstraite et pourra prendre des formes diverses en fonction du cadre théorique retenu, devra être capable d'engendrer la totalité des valeurs effectives de la préposition au travers d'un calcul effectué à partir des indices co-textuels et situationnels. On comprend ainsi que le traitement sémantique des prépositions dans les manuels soit toujours décevant. L'approche traditionnelle, telle qu'illustrée par CGEL 1985, se traduit par un émiettement des significations, avec un regroupement des valeurs spatio-temporelles, censées être plus facilement repérables, et une énumération disparate des autres valeurs. Le traitement grammatical n'est pas alors fondamentalement différent du traitement lexicographique, et en l'absence d'un modèle d'analyse global et homogène, reste le plus utile. L'approche localiste, telle qu'illustrée par CGEL 2002, ne peut être généralisée, en synchronie contemporaine, à l'ensemble des prépositions et se fonde souvent sur les propriétés des objets de l'extralinguistique plus que sur celles des unités linguistiques elles-mêmes ${ }^{24}$.

\footnotetext{
${ }^{22}$ C'est moi qui souligne.

${ }^{23}$ Voir par exemple Heine 1991.

${ }^{24}$ Sur ce point, v. Gilbert 2003, 37-41.
} 
On pourrait penser que l'étude du sens des prépositions est finalement hors du domaine de la grammaire. Ce serait oublier que la préposition assure l'intégration de son complément dans la phrase, et qu'à ce titre, ses propriétés sémantiques sont le plus souvent conditionnantes à l'égard à la fois du terme régi et du terme recteur. On peut faire l'hypothèse que l'emploi de toutes les prépositions, y compris les plus grammaticalisées - au sens où certaines ne semblent jouer qu'un rôle purement syntaxique, par exemple introduire le complément d'un verbe ou d'un adjectif - est contraint par leur sémantisme propre ${ }^{25}$. En d'autres termes, si decide se construit avec on (We decided on blue paint for the bedroom) et opt avec for (They opted for a chemistry course), c'est que chacun des deux verbes, pourtant proches sémantiquement, a une signification spécifique, qui sélectionne telle ou telle préposition, les deux significations, par interaction mutuelle, construisant in fine le sens de l'ensemble.

Une grammaire a forcément ses propres limites. Les grammaires traditionnelles ne s'appuient pas sur une théorie sémantique et sont condamnées à un répertoire, plus ou moins complet, d'effets de sens. CGEL 2002 a le mérite de faire appel à un cadre théorique spécifique, celui de la sémantique cognitive. Il est indéniable que l'approche cognitive a permis de renouveler l'étude des prépositions, mais la primauté accordée au domaine spatial constitue une hypothèse forte qui, dans l'état actuel des connaissances, n'est pas généralisable à l'ensemble des prépositions en synchronie contemporaine.

\section{La préposition et le poids de la tradition}

Au terme de ce panorama forcément limité - n'a pas été soulevé par exemple le problème des locutions prépositionnelles -, se pose la question de la nécessité d'une catégorie préposition dans une description grammaticale de l'anglais. Il s'avère que ni les approches traditionnelles, ni celles qui remettent en cause la tradition, ne sont à même de fournir une définition ou d'établir un ensemble de propriétés, syntaxiques et sémantiques, qui vaudraient pour toute la classe. Toute classification a ses limites liées à l'existence de tensions antagonistes dont le grammairien ne saurait se départir. D'un côté, il cherche à établir un système de généralisations en rassemblant dans une même classe des unités qui se caractérisent par des régularités de comportement; de l'autre la langue comporte une multitude d'unités ayant des comportements idiosyncratiques. D'où la nécessité d'effectuer des choix qui doivent être motivés. Les catégories fléchies (nom, verbe, adjectif), définies essentiellement à partir de traits morphologiques, font depuis longtemps l'objet d'un certain consensus. Le point faible de toutes les taxinomies a toujours été constitué par les mots indéclinables (adverbe, préposition, conjonction) et nous avons vu la difficulté qu'ont les grammairiens à établir des frontières entre ces trois catégories, si tant est qu'elles doivent être conservées toutes les trois. Concernant la préposition anglaise, ces tensions se traduisent par le fait que les grammairiens, qu'ils opèrent dans une optique traditionnelle ou non, proposent un classement fondé sur une catégorisation stricte (telle unité appartient ou n'appartient pas à la classe $^{26}$ ), tout en opposant au sein de la classe prépositions centrales et prépositions marginales, sans toutefois que le «centre » ne soit clairement défini. Opposer prépositions centrales et prépositions marginales, c'est reconnaître implicitement que la catégorie

\footnotetext{
${ }^{25}$ La notion de «préposition incolore ou vide», couramment utilisée en grammaire française (cf. Melis 2003, 83), n'appartient pas à la tradition grammaticale anglaise : faut-il y voir la reconnaissance implicite d'un sémantisme propre à chaque préposition de l'anglais ?

${ }^{26}$ Avec possibilité de double appartenance : like, due, near, etc. sont prépositions ou adjectifs (v. CGEL 2002, 608-610).
} 
correspond à un ensemble flou au sein duquel existent des degrés de «prépositionalité ${ }^{27}$ ». Parce que le souci de catégorisation est inhérent à l'entreprise grammaticale, rares sont les manuels qui se situent dans une perspective continuiste. CGEL 1985 constitue une exception et a fréquemment recours au concept de gradient (loc. cit., 90) mais sans l'utiliser explicitement pour son traitement de la classe prépositionnelle. Une approche consistant à établir la liste de tous les traits caractéristiques de la préposition centrale prototypique - sorte de " centre organisateur» de la classe - et à envisager chaque préposition en termes de distance relativement à ce centre, permettrait d'éviter la «raideur» qui caractérise les taxinomies proposées par les grammaires.

Les écarts par rapport à la tradition introduits par CGEL 2002 sont motivés par le souci d'intégrer les résultats des recherches linguistiques menées au cours des dernières décennies. La façon de traiter les prépositions montre néanmoins le poids de la tradition. Le terme même de préposition n'est pas remis en cause, bien que le périmètre de la classe soit considérablement élargi et que le mot perde son sens étymologique (du latin proe-positio $=$ putting before) puisque ce manuel admet l'existence de prépositions totalement intransitives ou post-posées à leur régime (notwithstanding the weather / the weather notwithstanding) ${ }^{28}$. Il est retenu en raison de son caractère établi et parce que le sens originel du préfixe pre- est largement oblitéré (loc. cit., 602). Les innovations sont présentées en contraste avec l'analyse traditionnelle, laquelle sert de point de départ et apparaît comme une sorte d'étalon à l'aune duquel il est possible de mesurer les écarts qu'elles induisent. Ainsi les propriétés de la classe sont initialement décrites à partir d'unités qui sont « uncontroversially prepositions » (p. 599), c'est-à-dire reconnues comme prépositions par la tradition. Ces unités, qui sont les seules ensuite à être envisagées sur le plan sémantique, sont, à plusieurs reprises (cf. p. 612), déclarées constituer le noyau dur de la classe, comme si les «conjonctions », pourtant recatégorisées sans ambiguité en prépositions, n'appartenaient pas pleinement à la classe. Mais il est vrai qu'un manuel de référence de cette ampleur s'adresse à un public large et doit pouvoir être utilisé par tous ceux qui s'intéressent à la langue anglaise, sans être forcément spécialistes de linguistique. A ce titre, le lecteur potentiel est marqué par des siècles de tradition grammaticale et il y a fort à parier que la doxa traditionnelle a encore de beaux jours devant elle, d'autant que si les avancées de la linguistique contemporaine sont incontestables, les principaux concepts grammaticaux, et tout particulièrement celui de préposition, sont encore loin d'être stabilisés.

\footnotetext{
${ }^{27}$ Tout comme on peut admettre l'existence de degrés de nominalité, d'adjectivité ou de verbalité (v. Cordier \& François éds., 2002).

${ }^{28}$ En vieil anglais, les prépositions «centrales » peuvent suivre leur complément si celui-ci est de nature pronominale : Tostig him to beah = Tostig gave allegiance to him $;$ Heom wi 才feaht Morkere eorl = Earl Morcar fought against him (Peterborough Chronicle 1066).
} 


\section{Références bibliographiques}

BIBER D. et al., 1999, Longman Grammar of Spoken and Written English, Harlow, Longman.

CERVONI J., 1991, La préposition. Etude sémantique et pragmatique, Paris/Louvain-laNeuve, Duculot.

CHERCHI L., 1986, La grammaire anglaise au fil des textes, Dijon, ALEI.

CORDIER F. \& FRANCOIS J., éds., 2002, Catégorisation et langage, Paris, Hermès.

FRIES C.C., 1952, The Structure of English, London, Longman.

DUFFLEY P., 1992, The English Infinitive, London \& New York, Longman.

GILBERT E., 2003, "Across, by et through. Considérations sur les conditions de représentation métalinguistique des prépositions", Anglophonia, 14, 37-61.

GILBERT E, 2004, "Ebauche d'une formalisation des prépositions in, on et at», L'identification, J.C. Souesme, éd., Cycnos, 21 (1), 93-111.

GREENBAUM S. \& QUIRK R., 1990, A Student's Grammar of the English Language, Harlow, Longman.

GROUSSIER M.L., 1997, «Prépositions et primarité du spatial : de l'expression de relations dans l'espace à l'expression de relations non-spatiales », Faits de Langue, 14, 221-234.

GUIMIER C., 1995-1996, "A study in grammatical semantics: the preposition to in English », Sigma, 17-18, 83-95

GUIMIER C., 1996, Les adverbes du français : le cas des adverbes en -ment, Paris/Gap, Ophrys.

HARRIS J., 1751 (1968), Hermes, of a Philosophical Inquiry concerning Language and Universal Grammar, Menston, The Scolar Press.

HEINE B., 1991, "From cognition to grammar - evidence from African languages" Approaches to Grammaticalization, vol. 1, E.C. Traugott \& B. Heine, eds., Amsterdam/Philadelphia, Benjamins, 135-142.

HUDDLESTON R.H. \& PULLUM G.K. et al., 2002, The Cambridge Grammar of the English Language, Cambridge, Cambridge University Press. (= CGEL 2002)

ILINSKI K., 2003, La préposition et son régime. Etude des cas atypiques, Paris, Champion. JESPERSEN O., 1924, The Philosophy of Grammar, London, Allen \& Unwin.

JESPERSEN O., 1933, Essentials of English grammar, London, Allen \& Unwin.

JESPERSEN O., 1940 (1965), A Modern English Grammar on Historical Principles, Part V, London, Allen \& Unwin and Copenhagen, Munksgaard (= MEG V)

LE GOFFIC P., 1993, Grammaire de la phrase française, Paris, Hachette.

MELIS L., 2003, La préposition en français, Paris/Gap, Ophrys.

MICHAEL I., 1970, English Grammatical Categories and the Tradition to 1800, Cambridge, Cambridge University Press.

MURRAY L., 1795 (1968), English Grammar, Menston, The Scolar Press.

NESFIELD J.C., 1898 (1961), English Grammar, Past and Present, London, Macmillan.

QUIRK R. et al., 1985, A Comprehensive Grammar of the English Language, London/New York, Longman. (= CGEL 1985)

SWEET H., 1891-1898 (1968), A New English Grammar, Oxford, Clarendon Press.

TESNIERE L., 1969, Eléments de linguistique structurale, 2nde éd., Paris, Klincksieck.

TREMBLAY M., 1999, « Du statut des prépositions dans la grammaire », Revue Québécoise de Linguistique, 27(2), 167-183.

TYLER A. \& EVANS V., 2003, The Semantics of English Prepositions, Cambridge, Cambridge University Press. 


\begin{abstract}
:
All grammars of the English language resort to a classification of words into 'parts of speech' or syntactic categories and none of them can do without prepositions. Even though the class seems to be well-established, a number of divergences appear in the way prepositions are dealt with. The main points at issue are discussed through a comparison of two major handbooks : Quirk et al., Contemporary Grammar of the English Language (1985) which represents the traditional approach to prepositions and Huddleston \& Pullum et al., Cambridge Grammar of the English Language (2002) which adopts a significantly different conception.
\end{abstract}

